\title{
Viral myocarditis and dilated cardiomyopathy - an experimental study with special reference to immunological behaviour of T-and B-lymphocytes
}

\author{
Chuichi Kawai and Chiharu Kishimoto
}

Kyoto University, Kyoto, Japan

\begin{abstract}
Summary: The direct transition from acute myocarditis to dilated cardiomyopathy has been demonstrated in an inbred strain of DBA/2 mice inoculated with the M-variant of EMC virus. The severity of myocarditis, which determines the development of congestive heart failure and, later, the diseased state resembling dilated cardiomyopathy, seems to be mediated by $T$-lymphocytes.
\end{abstract}

Our group (Kawai, 1971) and later, Cambridge et al. (1979) demonstrated significantly raised levels of antibody titres against Coxsackie B viruses in patients with cardiomyopathy. As a result, we assumed that there might be a relatively high incidence of past viral infections in cardiomyopathic patients. Electron microscopic examination, isolation techniques and immunofluorescent studies have failed to show direct evidence of virus infection in the human biopsied myocardium in patients with cardiomyopathy. Therefore, we concluded that the previous viral infection probably created an autoimmune process which led to progressive myocardial damage. However, even in experimental viral myocarditis, direct evidence to show the transition from acute viral myocarditis to cardiomyopathy has been lacking.

The purpose of the present paper is to show an animal model in which the direct transition from acute viral myocarditis to the diseased state resembling dilated cardiomyopathy can be demonstrated. Immunological behaviour of lymphocytes in the acute phase of viral myocarditis will also be discussed.

\section{Materials and methods}

Inbred strains of BALB/c mice and DBA/2 mice were inoculated with the M-variant of encephalomyocarditis (EMC) virus as described previously (Matsumori \& Kawai, 1982a, b). The B-lymphocyte was characterized by staining with fluorescein isothiocyanate (FITC)-labelled anti-mouse immunoglobulin; and the B-plus T-lymphocytes were stained with rat anti-Thy1,2 monoclonal antibody plus FITC-labelled anti-

Correspondence: C. Kawai, M.D., F.A.C.C. mouse immunoglobulin (Parish et al., 1974). The percentage of positive fluorescent cells in each specimen was determined by the examination of at least 200 cells.

\section{Results}

Fourteen days after inoculation of BALB/c mice there was dilatation of the ventricular cavities, pleural effusion, ascites and pulmonary congestion; the mice died of congestive heart failure. However, dilatation of the heart with or without hypertrophy had not developed in the chronic stage.

In DBA/ 2 mice, myocardial necrosis became extensive and mononuclear cell infiltration most prominent on day 14 after inoculation. Mice with severe myocarditis died of congestive heart failure in the acute stage of illness. In the mice with myocarditis that survived for 90 days, both the weight of the heart and the ratio of heart weight to body weight increased. The cross section of the heart demonstrated an enlarged left ventricular cavity with prominent myocardial fibrosis and hypertrophy. Mononuclear cell infiltration was not seen at this stage.

There was a marked decrease in the T-lymphocytes in the peripheral blood on day 13 after EMC virus inoculation in DBA/2 mice, when myocardial necrosis and cell infiltrations were most prominent. Thereafter, the T-lymphocytes increased again (Figure 1).

The T-lymphocytes in the myocardium showed reciprocal changes to those in the peripheral blood; almost all lymphocytes (approximately $80 \%$ ) infiltrated in the myocardium on day 10 to 13 were the T-lymphocytes, which were gradually replaced by the B-lymphocytes within 1 to 3 months (Figure 2).

The incidence of myocarditis inoculated with EMC 


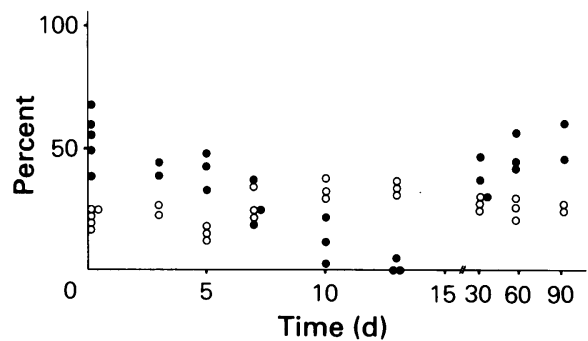

Figure 1 Behaviour of $(O) \mathrm{T}$ - and $(\mathrm{O}) \mathrm{B}$-lymphocytes in the peripheral blood in DBA/2 mice inoculated with the $M$-variant of EMC virus.

virus was equal in the nude mice, but the pathological changes in the myocardium were less prominent, and the death rate due to congestive heart failure was very low. In the spleen cell (B + T-lymphocytes) transferred nude mice, the death rate due to congestive heart failure increased.

\section{Discussion}

In this study, we have demonstrated that DBA/2 mice with viral myocarditis developed the lesions resembling those found in dilated cardiomyopathy in the chronic stage. The development of cardiac dilatation

\section{References}

CAMBRIDGE, G., MACARTHUR, C.G.C., WATERSON, A.P., GOODWIN, J.F. \& OAKLEY, C.M. (1979). Antibodies to Coxsackie B viruses in congestive cardiomyopathy. British Heart Journal, 41692.

KAWAI, C. (1971). Idiopathic cardiomyopathy: a study on the infectious-immune theory as a cause of the disease. Japanese Circulation Journal, 35, 765.

MATSUMORI, A. \& K̂KAWAI, C. (1982a). An experimental model for congestive heart failure after encephalomyocarditis virus myocarditis in mice. Circulation, 65, 1230.

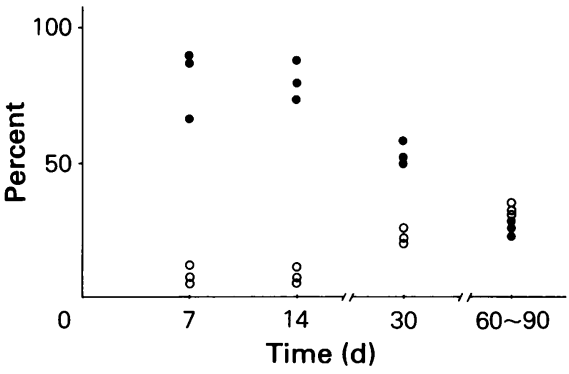

Figure 2 Behaviour of $(O) \mathrm{T}$ - and $(\mathrm{O}) \mathrm{B}$-lymphocytes in the myocardium in DBA/2 mice inoculated with the $M$ variant of EMC virus.

and hypertrophy seems to depend on the severity of myocardial lesions, which must be severe, but permit the animals to survive.

What then are the immunological determinants in the host animal to affect severity of viral myocarditis in the acute stage? The present study clearly demonstrated that the T-lymphocytes may play an important role to determine the severity of myocarditis, and thus the development of congestive heart failure and, later, progress to dilated cardiomyopathy. The immunological process in the chronic stage, when the transition from viral myocarditis to dilated cardiomyopathy may take place, remains to be elucidated.

MATSUMORI, A. \& KAWAI, C. (1982b). An animal model of congestive (dilated) cardiomyopathy: dilatation and hypertrophy of the heart in the chronic stage in DBA/2 mice with myocarditis caused by encephalomyocarditis virus. Circulation, 66, 335.

PARISH, C.R., KIROV, S.M., BOWERN, N., BLANDERN, N. \& BLANDERN, R.V. (1974). A one-step procedure for separating mouse T and B lymphocytes. European Journal of Immunology, 4, 808. 